\title{
A simplex model for layered niche networks
}

\author{
P. Fraundorf \\ Physics $\&$ Astronomy, U. Missouri-StL (63121), St. Louis, MO, USA
}

(Dated: July 11, 2018)

\begin{abstract}
The standing crop of correlations in metazoan communities may be assessed by an inventory of niche structures focused inward and outward from the physical boundaries of skin (self), genepool (family), and meme-pool (culture). We consider tracking the progression from three and four correlation layers in many animal communities, to five of six layers for the shared adaptation of most humans, with an attention-slice model that maps the niche-layer focus of individuals onto the 6-variable space of a 5-simplex. The measure puts questions about the effect, on culture and species, of policy and natural events into a common context, and may help explore the impact of electronically-mediated codes on community health.
\end{abstract}

PACS numbers: 05.70.Ce, 02.50.Wp, 75.10.Hk, 01.55.+b

Contents

\section{Introduction}

\section{Correlation layers and boundaries}

III. Simplex models of attention or resource focus

A. Advertising Example

B. Highway Safety Example

C. Applications in Social Psychology

D. Political Science Example

\section{Hypotheses to test}

A. Link to memetic evolution

B. Codes that redirect behavior

1. Mediated interaction as niche substitute

2. Cultural cartoons: Xenophobia \& the web

C. Level devolution from 6 Down

1. Confusing observation \& consensus

2. Confusing consensus \& belief

3. Confusing belief \& observation

D. Level building toward 6

1. Tracking soft correlations

2. Context on message scope

3. Complementarity: Bundling code \& organism perspectives

4. Newsgroups vs. chats

\section{Discussion}

\section{Acknowledgments}

A. Why these six, and only six, levels?

\section{B. Representations of the 5-simplex}

\section{References}

\section{INTRODUCTION}

Thinking on multiple scales of space and time is a crucial element of the move toward sustainability. Here we discuss quantification of a multi-scale view of organization among metazoan individuals, which draws from roots in physical representation theory $\stackrel{1}{\underline{1}}$. This may be useful, for example, as we explore the short-term impact of new technologies as well as the long-term window of opportunity for metazoan life ${ }^{2}$.

The approach here builds on recent findings in three convergent disciplines. The first of these is nanoscience, where chemistry, physics, biology, engineering, medicine, crime scene investigation, ethics, and complex system studies of emergence run together. The second is astrobiology, where Eric Chaisson's Cosmic Evolution ${ }^{3,4}$ (a natural history of invention with physical science roots) and Brownlee and Ward's Rare Earth ${ }^{5}$ intersect our distant past, and our distant future. The third is complex system informatics, where the code-based sciences (genetics, computer science, linquistics), thermal physics, journalism, networks and statistical inference join up. For the most part, however, independent of their technical context the ideas described should also be possible to check against the reader's everyday experience. Thus we won't belabor the technical roots except when necessary.

\section{CORRELATION LAYERS AND BOUNDARIES}

One way to inventory social complexity is to think of it as a layered network of niches occupied by individuals, as illustrated in Fig. 1. Here we define niche as a correlation through time between one steady-state system (e.g. an organism or heat engine), or more generally one excitation (e.g. a particle), and its environment. Niche network inventories in this sense can be applied to complex systems on layers ranging from elementary (e.g. particle state inventories in statistical physics) to complex (e.g. metazoan communities). Of course in the more complex cases: Operational definitions, ennumeration of alternate 


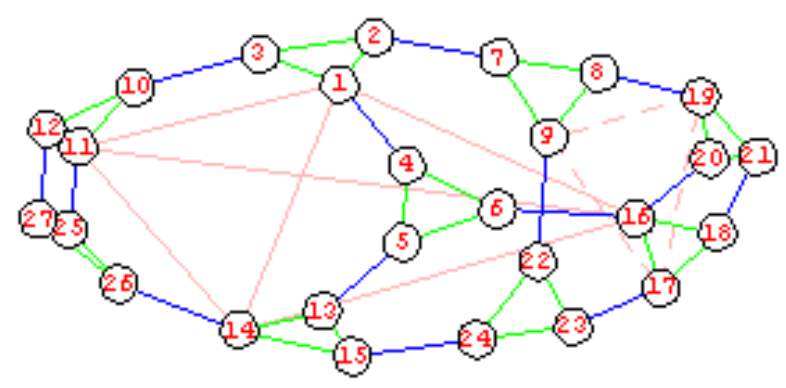

FIG. 1: Layered niche network inventory: This one shows four scales of correlation: self (\#), friends (blue), family (green) \& teams (light red).

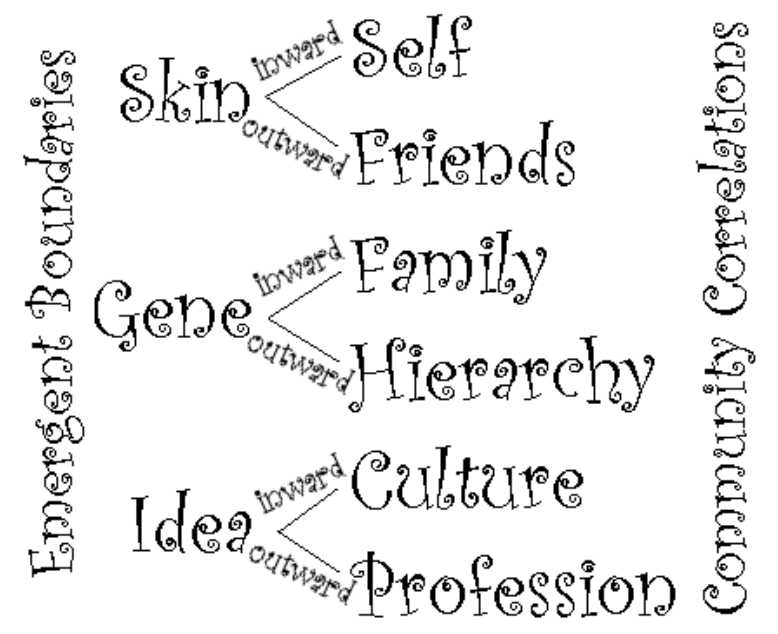

FIG. 2: Community correlation schematic

states, and objective implementation may be quite challenging, particularly if one wishes to put the resulting measures into $2^{\text {nd }}$ Law terms.

Returning to the community focus of this paper, the layers of a network built up from the level of individual metazoans are often conceptualized as inward- and outward- looking with respect to three increasingly larger physical boundaries, namely your skin, your family's gene pool, and your culture's idea pool. The pattern is illustrated in Figure 2. In other words, our social fabric is built on everyone's ability to take care of themselves, and be responsible to their friends, family, job, culture, and knowledge base.

The extent to which citizens can fill niches on all six of these levels is a measure of community complexity. The impact of policy decisions and disasters on community health may also be reflected by changes in the average number of niche levels (0-6) that each citizen can participate in. Increasing literacy, for example, likely improves a person's ability to contribute to the knowledge base with their own observations. Conversely, loss of one's home to a flood might wreak havoc on long term friendships and jobs, even if everyone's health and family stays

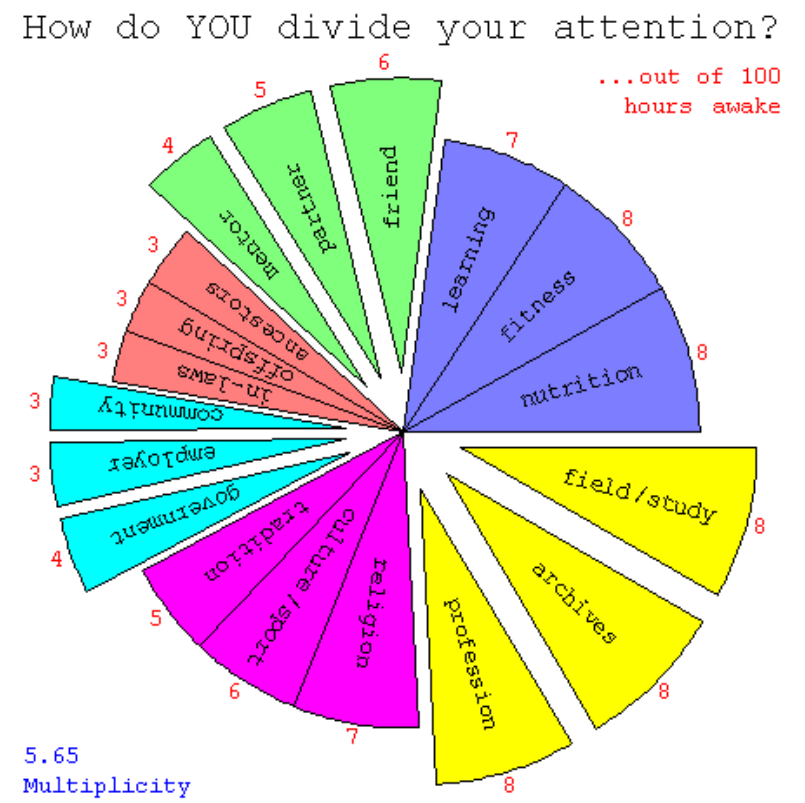

FIG. 3: Attention fractions for a hypothetical individual

intact.

Although an objective inventory of such niche occupancies is difficult (i.e. it is not easy to take the essence of a community and project it onto a spreadsheet), the spirit of a program which seeks to give everyone a chance to simultaneously take care of self, friends, family, hierarchy, culture and profession is well-grounded in our knowledge of simpler (e.g. physical) systems. It may also be satisfying in other ways. Thus for the sake of future discussion, we consider the ability of members to occupy niches on all six scales of organization as a measure of community health.

\section{SIMPLEX MODELS OF ATTENTION OR RESOURCE FOCUS}

To quantify, one might develop technical definitions for the above concepts (e.g. friendship as a pair correlation, political consensus as multi-family agreement, scholarly reports as codes that mirror nature, etc.). These technical definitions (like that for work in physics) could serve as a complement to their more diverse vernacular forms. From that point, one might then associate with each of $i=1, N$ individuals that fraction $f_{i j}$ of their attention allocated to maintaining correlations on each of the $j=1,6$ niche scales, as discussed in Appendix A For example, someone who spends directs all of their energy toward church-activities or dance-class (i.e. developing cultural connections) might have a larger value of $f_{i 5}$ than someone who spends all their time doing applied mathematics. The six $f$-values for an individual thus describe the distribution of size scales on which they connect to the community around (cf. Fig (3). 


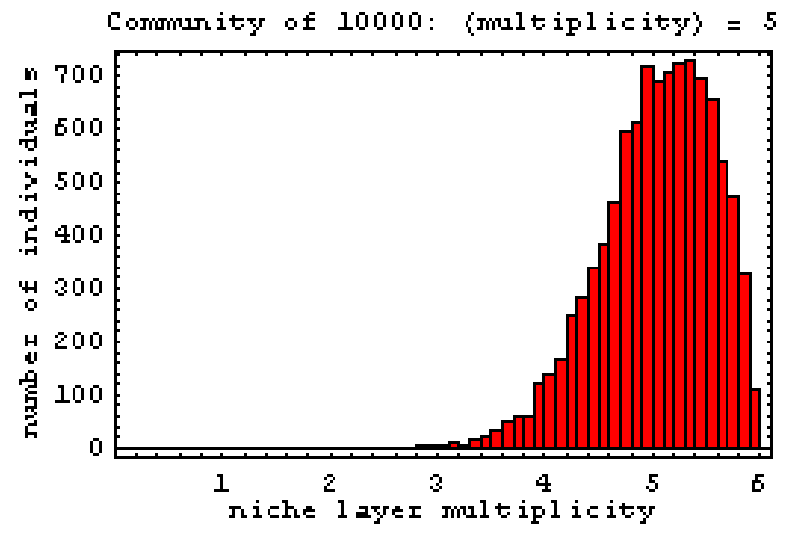

FIG. 4: Distribution of niche level multiplicity for community of 10000

In addition to the distribution of individuals in this 6 -parameter space, the average surprisal $s$ (in bits) of an individual's focus given only the $f_{i j}$ is one measure of level diversity for a community as a whole. Niche level multiplicity defined as $2^{s}$, a number between 0 and the active number of niche layers, allows us to put this measure of community health into even simpler terms.

The distribution of niche-level multiplicity for individuals in a community of 10000 is illustrated in Fig. 4. Here the attention fractions for each individual were chosen by assigning a random number between 0 and 1 to each, and then normalizing the set by dividing by the sum. This ad hoc protocol increases the likelyhood of small fvalues over that of a uniform assignment in f-space. It's as if each niche layer were assigned a stand-alone priority, later put into context by the fixed amount of time in a day.

The resulting niche-level multiplicity of the community as a whole (a geometric average of individual multiplicities) in Fig. 4 is five out of six. Potentially interesting questions include the following: In which column of this plot would you fit? How will your niche level multiplicity change during the next decade? How would similar plots look for the various communities of which you are a part? How will changes in the environment and/or economy affect the profile for those communities?

Since the six f-values for an individual are positive numbers which sum to one, they span the hypervolume of a unit-vertex 5-simplex in f-value's $6 \mathrm{D}$ parameter space, as discussed in Appendix B. Ternary sums which span this space (like $f_{i 1}+f_{i 2}+f_{i 3}, f_{i 4}, f_{i 5}+f_{i 6}$ ) thus plot as points in an equilateral triangle, while quaternary spanning sums (like $f_{i 1}, f_{i 2}, f_{i 3}, f_{i 4}+f_{i 5}+f_{i 6}$ ) plot as points in an equilateral tetrahedron. Individual states, without sum degeneracy, can also be plotted as point pairs in a set of vertex-joined tetrahedra (cf. Fig. 51). Properties of simplex models applied to other natural systems ${ }^{6}$ may prove useful here as well.

\section{A. Advertising Example}

The niche layer for self-care might be used to distinguish folks who select coffee, tea, or neither when given a choice. One could experimentally model the effect of ads on (i) the probability $f_{i 1}$ that folks will concentrate on self-care, and (ii) in so doing choose either coffee or tea. Thus we are looking at niche occupancies, and their correlation with specific idea sets. Advertisers are already doing this, even if not in this multiscale context.

\section{B. Highway Safety Example}

To maximize safety, automobile drivers should probably divide their attention between keeping their own vehicle safe, and not endangering others on the roads around. Simultaneous focus on other niche-level responsibilities while driving in this context serves as a distraction. Such distractions can move into real time when one is also holding and talking on a cell phone. Measurements of the resulting attention loss might be put onto a more quantitative footing using the conceptual tools discussed here.

\section{Applications in Social Psychology}

Control systems theory in sociology looks at the way that humans interface to the world through niche-related ideas. Although these ideas differ from one culture to the next, the multiscale or layered-network structure discussed here offers an integrative thread. For example, all human cultures distinguish between family and nonfamily individuals even if the word associations used are different. Via connections like this, data generated e.g. by affect control experiments in social psychology ${ }^{7}$ may be used to monitor the effect of media events or social policy on objective measures of community health. If we focus on the way that behavioral resources (rather than perceptual attention) are directed toward community correlations, this same integrative strategy may also be put to use in studying the behavioral ecology of animal communities centered more around genetically rather than culturally-transmitted codes.

\section{Political Science Example}

Here, let's select the social hierarchy layer and ask about the effect of ads on (i) the probability $f_{i 4}$ that folks will take political responsibility in a given election, and (ii) in so doing choose one party or another. Simulations over many decades here likely show some interesting oscillations. Again media is already being put to use by politicians, and so a developing awareness of the effect of those actions on community health may be a good thing. 


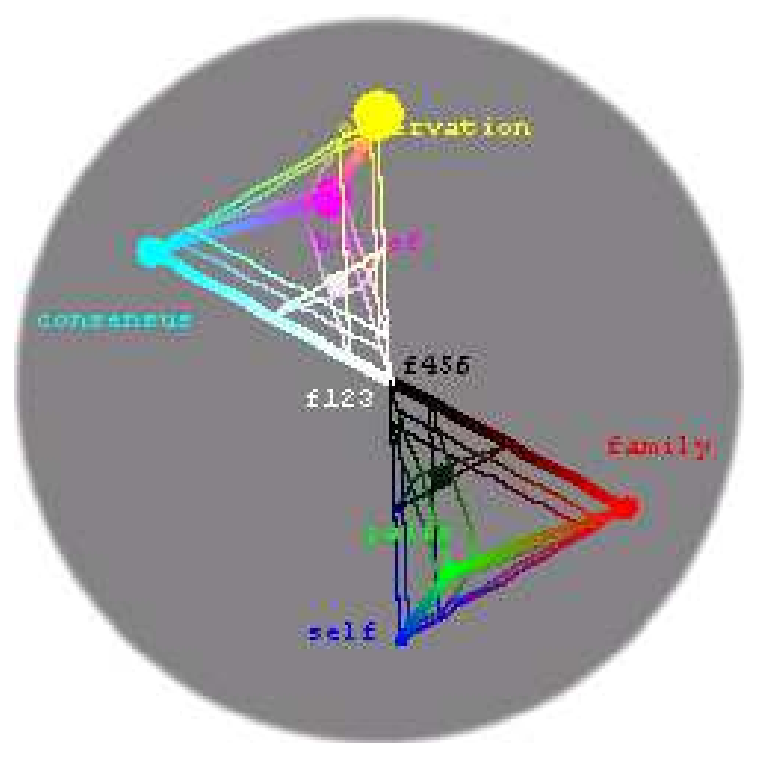

FIG. 5: Dual simplex representation (cf. Appendix B) of a $(1 / 6,1 / 6,1 / 6,1 / 6,1 / 6,1 / 6)$ niche assignment.

\section{HYPOTHESES TO TEST}

Given a measure of community health (niche level multiplicity) referenced to three physical boundaries (skin, family gene pool, and cultural idea pool), we now consider hypotheses that data on this measure might allow us to test. The focus will be on applications in human communities, and in particular the relationship of these measures to ideas (i.e. concept sets in use) and their means of replication.

The relationship between ideas and niche focus is of practical interest for two reasons. First, along with behavioral observations 8 and population surveys, communication traffic may provide important clues to the niche focus across a community. Secondly, mediated communications have become increasingly important as a mechanism for manipulating the public's attention, particularly by those with commercial and/or political interests. Multiscale thinking, with deep roots in the natural sciences, could offer a much needed public health perspective on these activities.

\section{A. Link to memetic evolution}

Before man's development of written communication, the separation between one's cultural knowledge and one's knowledge of nature's behavior was probably less well defined. Local culture was the source of both types of knowledge $\mathrm{e}^{9}$. In communities of animals with less developed oral communication skills, cultural training itself seems to be less developed but certainly not absent ${ }^{10}$. Thus it may be that the pre-history shared by all humans involved adaptation of individuals to five, and not six, layers of niche development in parallel with our shared refinement of oral communication skills.

This is important, because adaptation to five niche levels may be something most humans have in common. As humans face more complex challenges to their large population in days ahead, understanding what we can and cannot count on may be important. Advertisers and politicians are already putting their knowledge of in-born human responses to work on us.

Before discussing possible impacts of these in-born adaptations on community health, it's worth pointing out the recent philosophical interest in the "selfish" evolution of cultural codes 11 . The integrative context discussed here can provide reality checks from the physical sciences (e.g. the non-locality of mutual information in physical systems is not inherent in vernacular uses of the concept), and as we'll see may also connect it (ala McLuhan ${ }^{12}$ ) quantitatively to technology through representational systems running from language through print to the internet. The context of idea technology naturally, then, leads us to questions about the opportunistic role of human-developed codes as an antagonist to robust niche development, and following that to prospects for giving as many humans as possible a chance to fill niches on all six scales of organization.

\section{B. Codes that redirect behavior}

By associating replicable codes with elements of our environment (including other organisms), ideas serve to redirect human behavior much as greeting behavior in animals serves to redirect intra-species aggression ${ }^{13}$. However, in addition to helping us maintain levels of organization in human communities, un-informed "reduction to code" can also cause trouble. This is especially true now that ideas can be replicated electronically across the planet's surface in seconds, by anyone with access to a web cafe. Put another way, self-consciousness about our choice of ideas may be crucial. This is especially true of news media, when they insist that someone answer a particular question without justifying why that particular question is appropriate.

Here we discuss hypotheses associated with technological redirection of niche-forming behaviors. These include the web's ability to amplify a neolithic survival trait (inter-cultural xenophobia) into oscillations of destructive cartoonification, and more generally ways to minimize the bad effects of opportunities created as technology evolves.

\section{Mediated interaction as niche substitute}

If one recognizes money as a technology developed along with the development of food production and distribution, then one can also see that one of our oldest "professions", prostitution, arose as a way to technologically fake some rewarding aspects of a reproductive part- 


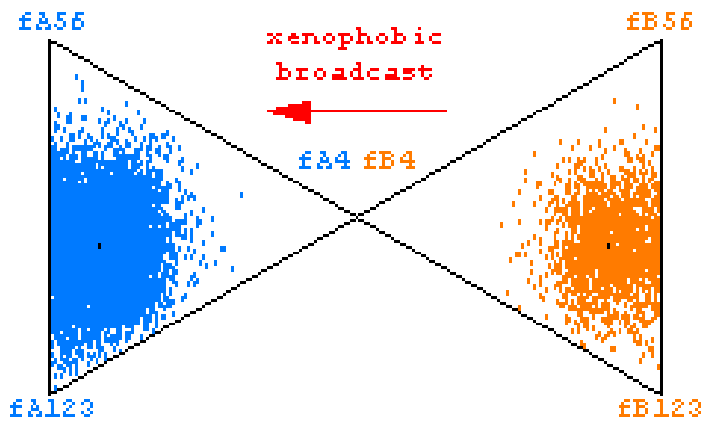

FIG. 6: Mediated f-value interaction between two communities

nership with help from a once new technology (namely, the coin of the realm). Any technology that redirects behavior away from responsible interaction with other humans plays the same role.

Just as money has its good side and its bad, so newer technologies (like video games) capable of distracting you from human interaction likely have positive uses e.g. in education, or as occasion for mental, physical, or team skills exercise. Hypothesis one, then, is this: Emphasizing the importance of responsible niche participation on each of the levels discussed above might help folks keep their eyes on the positive (rather than the "fake niche") aspect of these technologies, and in the process increase rather than decrease niche multiplicity.

\section{Cultural cartoons: Xenophobia \& the web}

Our evolved response to the idea of a boogeyman, if we ignore the dynamics of this idea, allows it to run amok in the information age outside of video games as well as in. For example if you respond to someone calling you subhuman by calling them subhuman, then the idea of treating others as subhuman is given a boost by both of you whether or not that behavior is in anyone's interest.

This is illustrated in the ternary plots of individual $f$ values for groups A and B in Fig. 6, where a resource problem that might only be solved by scientific observation $\left(f_{i 6}\right)$ precipitates discomfort that results in a xenophobic broadcast from one group of 2000 individuals (B). Because in today's world that broadcast is accessible to all, this broadcast can shift the focus of both the uncomfortable group (B), and the object group (A) of 10,000 individuals, toward political action $\left(f_{i 4}\right)$.

Electronic media capable of sharing broadcast and counterbroadcast may thus shift the focus of both populations away from where it is needed. Note that movement of individual dots in the figure toward the $f_{i 4}$ vertex denotes a shift of attention toward politics, regardless of the side they take on any given issue. If we recognize in this the dynamics of codes that had survival value long ago but can be improved upon now, then we have a better chance to keep such oscillatory cartoonification from causing more damage than necessary.

Hypothesis two: Such analyses might predict the toppling of each district's statues after deforestation on Easter Island ${ }^{14}$, or the electronic ramping of intensity in current wars by humans not thinking about the dynamics of xenophobic ideas in a resource-limited world. This is a special case of the ability of electronically-broadcast ideas to redirect the attention of large subsets of our population via evolved human perceptions. To the extent that mirror neurons $\frac{15}{5}$ can directly elicit feelings via the faux intimacy of an electronic broadcast (as with the "neighborhood gossip" ambience of some political attack ads), one can even imagine feedback loops that manipulate the public's attention on visceral levels to bypass logic and self-interest altogether.

\section{Level devolution from 6 Down}

We discussed earlier how our evolved response to language likely involved mainly niches pertaining to self, friends, family, hierarchy, and culture. This might help explain why some of us substitute our profession (if we find opportunity to develop one) for one of these other elements in our life.

One practical consequence: level blurring from 6 down to 5 increases the likelyhood of extremist niche occupancies, i.e. individuals whose behavior is informed to only a minor subset of the constraints associated with a 6 -level niche structure. For example, if one plots contours of constant net surprisal (separated by 0.1 bit) with respect to an equal-fraction ambient (where all fractions are $1 / \mathrm{N}$ ), when two levels become one those contours move outward toward the corners of the plot. If extremist preoccupation (the ignoring of correlations outside of one corner) helps replicate an idea which drives this blurring process, such blurring could easily be amplified in this age of electronic communications with no conscious input on the part of the participants.

When free energy per capita decreases, Eric Chaisson's anecdotal observations of the relation between complexity and free energy rate density suggest that complexity will also decrease, and thus that pressure to devolve civilization from 6 back down to 5 layers of individual correlation may increase. We mention three examples of this here.

\section{Confusing observation $\mathcal{E}$ consensus}

The scientific tradition involves observations of nature, faithfully reported, followed by the iterative refinement of conceptual models for its understanding and prediction. Social consensus about those models has its uses, but is generally a dated and belated spin off. Scientific observation is the horse which pulls that cart.

Similarly teaching science is about training each of us as critical observer, not about teaching the consensus. 
Thus when scientists and creationists argue about which consensus to teach, they are helping to blur the distinction between science and politics. Their discussion generally does not involve what observational tools to use. Thus a mistaken idea of science as "taught consensus" has folks fighting for no good reason. Once again our gut level reactions, in the face of ideas with electronic wings, are distracting us from the real challenges at hand. These challenges include decreasing free energy per capita that could help drive the confusion.

\section{Confusing consensus 85 belief}

The separation between church and state is an old story, with new chapters being written every day. We portray the drive to merge the two here in a different light, namely as community simplification (devolution) e.g. toward an endpoint where the alpha-wolf's majority decides on the ideas used. In effect this leaves room for fewer cultural niches than there are citizens, and points toward a memetic monoculture with shortcomings (as far as survivability is concerned) similar to those of genetic monocultures in biology. On the contrary, layered niche models suggest that consideration of endangered cultures may be as important scientifically as consideration of endangered species, if only we can separate science, culture, and politics in addressing it.

\section{Confusing belief $\&$ observation}

In the historical scheme of things the distinction between niches focussed on belief and observation is probably most recent, and hence also the one we've had least time to evolve with. As many have said, of course, there is no a priori reason why belief and observation should be in conflict unless one seeks to promote beliefs that fly in the face of observation. The good news from the other direction is that this scientific approach to correlationbased complexity protects cultural beliefs as an element of structure as zealously as it does the fruits of scientific observation. Simplifiers in either direction are where the real confusion lies.

\section{Level building toward 6}

We've talked a bit above about ideas which replicate easily among humans in an electronic age, but that also break down community correlations in the process. We hypothesize here a few strategies that might help to strengthen correlations instead.

\section{Tracking soft correlations}

Rather than concentrate only on economic measures and population (which are relatively easy to be objective about), consider also trying to track niche-layer occupancy per citizen (0-6) in a community as a function of time. Why? One reason is that it at least tries to register the impact of policy changes on deeper aspects of community structure. Although objective quantification complicated, since even the participants may not be sure how well they are doing, this is a place where technology might be applied to social health as it is already applied to the health of individuals. As economists look for measures of progress linked to the steady state rather than to rates of growth, this might be useful there as well.

\section{Context on message scope}

Although it is flattering to be asked one's opinion about all manner of subjects, the fact is that we have a mandate and resources to be informed about some subjects more than others. For example, a company manager's (or policy's) goals and effectiveness will look different from the vantage point of: (i) a company employee, (ii) one of the company's customers, and (iii) the company's board of directors. That's because the information environment, as well as the mandate, of each of these parties is quite different. Each input may be relevant to informed evaluation. Similarly by using non-technical vernacular, science popularizers often ruffle the feathers of their more technically particular colleagues even though they are much more fun for the general public to listen to.

In both cases, tagging messages with information on (i) their source, and (ii) the intended scope of their audience, could help. As with food tagged according to source and intended destination, and with political messages bundled with a candidate's admission that they endorse it, such accompanying information can mediate one's visceral reaction to the message (or meal) by prompting them to ask: Is the sender informed to what I should be thinking about, and is my reaction to it part of a larger context that I want to support or not? What layers of organization, and what populations, are being served thereby? Such information puts messages into a multiscale context.

\section{Complementarity: Bundling code $\&$ organism perspectives}

Get in the habit of talking about code plus organism perspectives when reporting news, and use idea codes for their modern relevance rather than only because they appeal to our prehistoric selves. For example, when two people get into a fight after the exchange of "inappropriate gestures" toward one another, in addition to report- 
TABLE I: Boundaries for social organization in metazoan communities

\begin{tabular}{c|c|c|c|c} 
Inward Focus & Physical Boundary & Outward Focus & Applies To & Correlation Model \\
\hline Individual & Metazoan Skin & Env/Pairs/Friends & All & Patterns in SpaceTime \\
\hline Family & Gene Pool & Consensus Hierarchy & Animals & 4-scale resource slicing \\
\hline Culture/Beliefs & Meme Pool & Professional Observation & Idea Sharers & 6-scale attention slicing
\end{tabular}

ing the human spectacle the reporter might examine the history of such gestures (which in related form even occur among non-human primates in the wild). There are other places where organism behavior is not the whole story.

The discussion of Fig. 6 is a case in point. If the press in region $\mathrm{B}$ describes a protest group in region $\mathrm{A}$ as the enemy, an oscillation of the sort mentioned above might start in the absence of opposition to begin with. That is, in this information environment an oscillation might nucleate from random fluctuations and be amplified into larger opposing pushes while the underlying resource problem falls off the radar. Linking the politics $\left(f_{i 4}\right)$ to religion $\left(f_{i 5}\right)$ or family $\left(f_{i 3}\right)$ might increase the amplitude of excursions toward the political corner of the niche-focus simplex, thus increasing extremism by lessening the pull toward other corners. Thus the media of this example might play an unwitting role via its critical focus on organisms but not ideas.

A global electronic media ignoring replicable code dynamics and limitations, but focused on pushing buttons as cheaply as possible, could thus be doing more harm than good. This leads to the hypothesis that yellow journalism can negatively impact community health quantitatively, in terms of niche-layer multiplicity.

\section{Newsgroups vs. chats}

Finally, instant messaging and chat rooms naturally represent their participants as replicable code, and have a reputation for eliciting "flame wars" between participants. These is another case of technologically redirected niche-forming behavior, since they take place in a world of replicable codes, and they can and are turned off at the flick of a switch much like a video game battle when it's time to go to bed.

However technology also has potential for archiving responsible interaction, and for saving correlations for access by future generations. Usenet newsgroups, recently hooked into browsers and cloned in moderated-access form by Google, are a case in point. Here, niches of accountable interaction on many levels can be developed, nurtured, and later studied. Newsgroup work for over a decade by John Baez on current finds in mathematical physics is a case in point. These mediated streams allow one to iteratively develop healing ideas for a community of interest, and to simultaneously document the process for participants downstream.

\section{DISCUSSION}

Thus correlation inventories with roots in the physical sciences may prove useful in monitoring the state and process dynamics of layered niche networks, including the metazoan communities of which we are a part. Other interesting features of this approach include the observation that idea sets (e.g. values) targeted toward the maintenance of specific levels of correlation in such inventories are easily identified, and that the stone age history that most humans share $\underline{\underline{9}}$ likely adapted us primarily to deal with only five of these six levels.

We outline a simplex model based on the focus of attention among metazoans toward the buffering of community correlations looking inward and outward from the edges of body, family, and culture. Anecdotal data, obtained largely from communication traffic particularly in human communities, suggests a number of interesting applications for this model. However quantitative behavioral observations, population surveys, and data on communication focus as well, are signficant hurdles at this point to putting the model to use.

\section{Acknowledgments}

I would like to thank Eric Chaisson at Tufts, Peter Rogan at UM-KC, Edwin Taylor at MIT, Tom Schneider of NIH, Don Brownlee at U. Washington, and the late E. T. Jaynes at Washington University for many interesting papers and discussions.

\section{APPENDIX A: WHY THESE SIX, AND ONLY SIX, LEVELS?}

Recognizing correlations between systems on different size scales probably requires that we know where a given system starts and ends, or in other words a definition of boundaries. In the case of complex systems, their boundaries generally start out as ill-defined but over time emerge as worth taking for granted.

Examples of this over time include the development of a star from a diffuse cloud of gas, the formation of planets from a bunch of tiny dust particles in orbit, the development of predictably structured enzyme-molecule surfaces from mixed atoms in a random soup, the formation of bilayer cell membranes with highly sophisticated pore structures to facilitate reproduction of useful biochemical cycles, the development of skin-bound meta- 


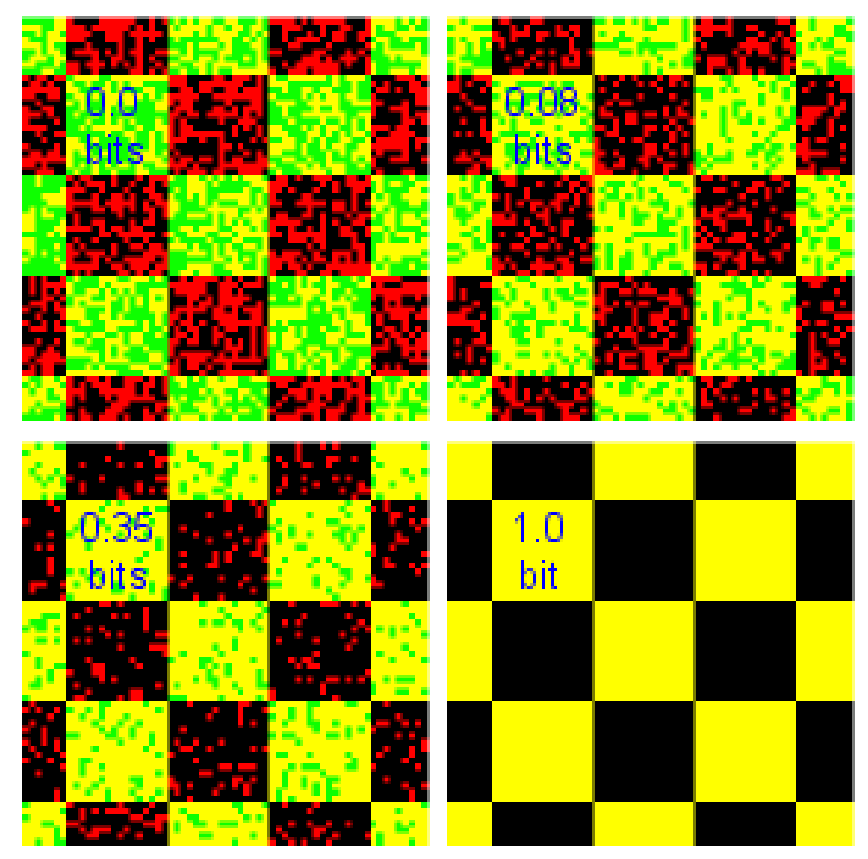

FIG. 7: Geographic correlations in a population

zoans with fancy endocrine, nervous, gastro-intestinal, circulatory, and immune systems from simpler tissue alliances like those found in jelly fish, etc. In each case, the new boundary arrives out of the blue and in poor focus. For a finite time, however, these boundaries begin to take on clear and practical significance. Each new boundary, in turn, is a jumping off point for emergence of the next boundary in our tree of layered correlations (cf. Table I here $\left.\frac{16}{}\right)$.

On size scales up from that of multi-celled organisms suppose that we consider only three such boundaries, namely metazoan skins, molecular code-pool boundaries (already complicated in a geometric sense), and idea code-pool boundaries (e.g. as physically encoded in recordable speech, writing, and a wide range of replicable digital formats including video). At first glance it seems that plants correlate with their environment spatially (cf. Fig. 7), but otherwise act more as heat engines (which capture thermodynamic availability) than as information engines (which buffer the kinds of code-pool correlation discussed here). For example, what plants show bias toward their own weaned offspring?

On the other hand, along with self-care animals seem comparatively active at pair interaction, as well as in the buffering of correlations directed inward (family) and outward (hierarchy) from their immediate gene pool. Activity focused inward and outward from skin and gene pool can be represented by the tetrahedral 3-simplex shown in Fig. 8. The investment of most animals in correlations directed inward and outward from cultural boundaries, however, seems pale in comparison to the attention directed by humans. In order of increasing layerscale the three boundaries (Table @) thus give rise to six
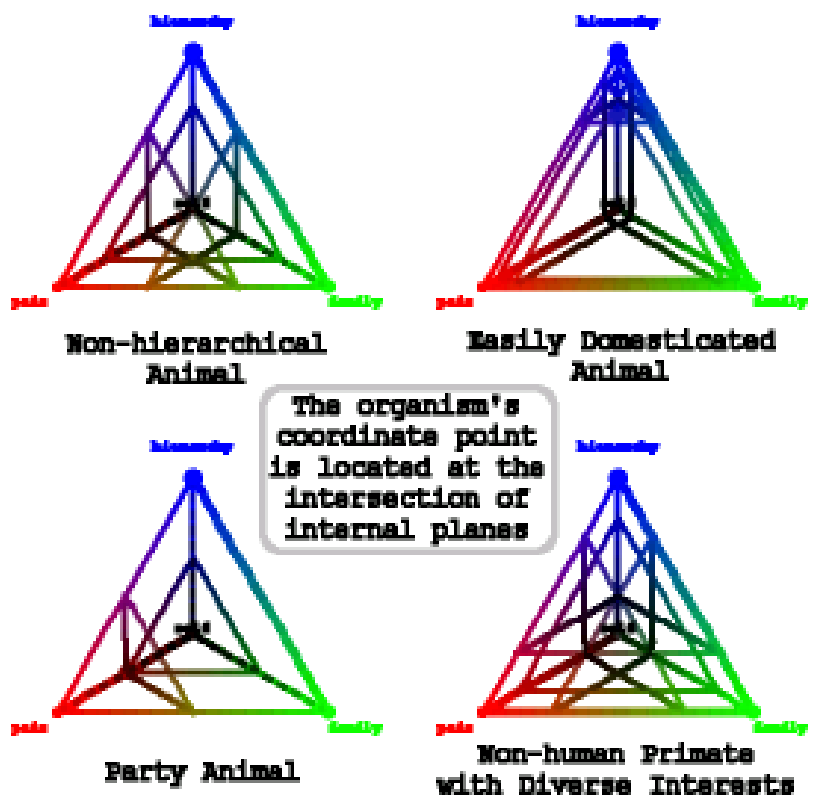

FIG. 8: Limiting cases of resource-focus on the 3-simplex with foci looking inward and outward from skin (self-pair) and gene-pool (family-hierarchy). The "party animal" assignment (sans negative connotation) in the lower left corner, namely $1 / 2,1 / 2,0,0$, refers to an organism invested in friendships and self, without regard to elements of the larger picture. It might also characterize youngsters of a species who've not yet shouldered the responsibilities of an adult.

very popular subjects of human discourse, namely health care, pair bonds, family matters, political hierarchy, culture/religion, and scientific/extra-cultural lore.

In even more basic physical terms, the magic of complex systems comes from the correlations which make the whole more than a sum of parts. This is the mathematical definition of mutual information. Niches that target the nurturing of correlations of the above 6 types (e.g. intra-family or inter-family) might comprise much of that magic in communities. In principle one could directly measure the effect of behaviors on correlations of this sort, like the spatial correlations between red organisms and environmental greenery in the above figure at right (recall that red PLUS green equals yellow). However, the dynamical nature of layered network correlations, as well as the problems that plague measurement of algorithmic complexity in computer programs (e.g. we are likely to miss important emergent patterns), make this an incomplete solution. We therefore suggest asking for each individual: "What fraction of their attention and/or effort is targeted toward the buffering of subsystem correlations in each of these 6 areas?" These are the $f$-values mentioned in Section III.

This "attention-slice" strategy for quantifying correlations in animal communities thus builds on recognition of two kinds of thermodynamic symbiosis. The first kind 

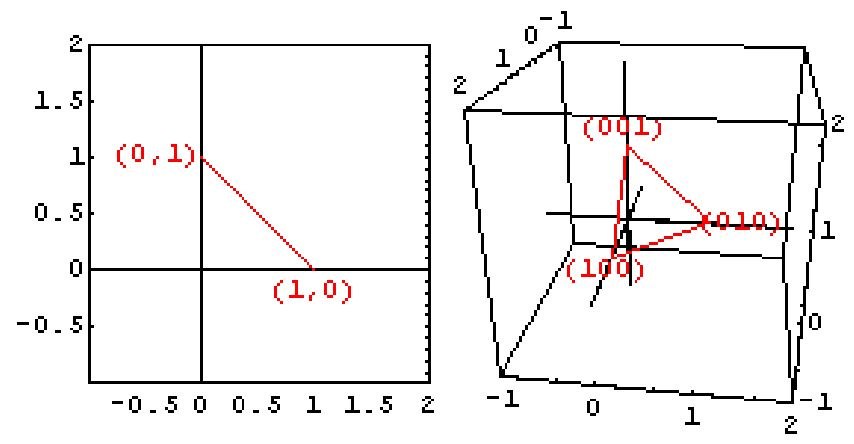

FIG. 9: Simplex mapping of 2 and 3 probabilities

is the informatic symbiosis between steady state excitations and replicable codes, integral to life of all sorts. For example, microbes store information on how to make useful proteins in nucleic acid codes whose perspective is as important as that of the microbes that carry them. Similarly, governmental hierarchies store information on useful procedures in books of law which carve out their own evolutionary tale.

The second kind is the trophic symbiosis between multi-celled plants and animals, wherein the former (autotrophs) convert thermodynamic availability (for the most part from sunlight caught by leaves facing skyward) into available work which in turn is thermalized by the latter (heterotrophs) while constructing their layered networks of subsystem correlation. The latter correlations include lively friendships, family activity, team accomplishments, cultural traditions, as well as libraries of observational data (with unavoidable interpretation) on how the world around works. No surprise then that absence of these traits is sometimes compared to a vegetable-like existence.

\section{APPENDIX B: REPRESENTATIONS OF THE 5-SIMPLEX}

If you have $N$ numbers that are positive and add up to one, then all possible values of those numbers plot within a finite $N-1$ dimensional figure. That's because the locus of such points in $N$-dimensional space is the $N-1$ dimensional analog of a triangle (i.e. an $N-1$ simplex). For example, the composition of a binary solidsolution series can be represented by a straight line (1simplex) that runs from $(1,0)$ to $(0,1)$. The composition of a ternary mixture in 3 -space falls within a flat $2 \mathrm{D}$ triangle (2-simplex) with vertices at $(1,0,0),(0,1,0)$ and $(0,0,1)$. Figure 9 illustrates how each of these structures is embedded in the higher dimensional space of which it is a part.

The 3D locus in four-dimensional space of a quaternary mixture is, similarly, a tetrahedron (3-simplex) with vertices at $(1,0,0,0),(0,1,0,0),(0,0,1,0)$, and $(0,0,0,1)$. Higher dimensional spaces work the same way, although they (and their embeddings) are harder to visualize. Probabilities as well as compositions are generally positive numbers that, taken together in a complete set, all sum to 1 . Thus $N$ probabilities can also be mapped to the space within an $N-1$ simplex.

A useful feature of these simplex mappings is the ability to project to lower dimension simply by labeling one vertex as a sum of probabilities. This works because the sum of a subset (say $m$ ) of $N$ normalized probabilities is simply another probability. This sum, in turn, when combined with the $(N-m)$ non-subset probabilities, continues to add to one. While the original $n$ probabilities are represented by an $(N-1)$ simplex, the new $(N-m+1)$ probabilities map to an $(N-m)$ simplex for easier visualization. Of course, this projection process also throws away information.

To avoid this loss of information, one can use the subset sum (of $m$-probabilities above) as a "dipole separation vertex". For the case when $N=6$ and $m=3$, each set of probabilities can then be represented by a pair of dots on parallel planes of fixed separation. The planes reside in vertex-to-vertex three simplexes, as shown is Fig. 5. The choice of the dipole-separation subset (e.g. use of $f_{i 1}+f_{i 2}+f_{i 3}$ versus $f_{i 1}+f_{i 3}+f_{i 5}$ ) affects which patterns are highlighted. This symmetry breaking, of course, may be an advantage if the system of probabilities being visualized has a matching asymmetry.
* pfraundorf@umsl.edu

1 C. M. Lapilli, P. Pfeifer, and C. Wexler, Phys. Rev. Lett. 96, 140603 (2006).

2 P. D. Ward and D. Brownlee, The life and death of planet earth (Copernicus, New York, 2000).

3 E. J. Chaisson, Complexity 9, 14 (2004).

4 E. J. Chaisson, BioSystems 46, 13 (1998).

5 P. D. Ward and D. Brownlee, Rare earth: Why complex life is uncommon in the universe (Copernicus, New York, 2000).

${ }^{6}$ H. Ohtsuki and M. A. Nowak, J. Theor. Biol. 243, 86 (2006).

7 C. A. Francis, in Purpose, meaning and action: Control systems theories in sociology, edited by K. A. McClelland and T. J. Fararo (Palgrave MacMillan, New York, 2006), pp. 139-162.

8 H. K. Reeve, in Model systems in behavioral ecology, edited by L. A. Dugatkin (Princeton University Press, New York, 2001), pp. 57-71.

9 J. Diamond, The third chimpanzee: The evolution and future of the human animal (Harper Collins, New York, 1992).

10 C. van Schaik, Science 299, 102 (2003).

11 K. Distin, The selfish meme (Cambridge University Press, New York, NY, 2005).

12 M. McLuhan, The Gutenberg galaxy: The making of typo- 
graphic man (publisher, address, 1962).

13 K. Lorenz, On Aggression (Harcourt, New York, 1966).

14 J. Diamond, Collapse: How societies choose to fail or succeed (Viking/Penguin, New York, 2004).
15 G. Rizzolatti, L. Fogassi, and V. Galese, Scientific American 295(5), 54 (2006).

16 P. Fraundorf, accepted for Complexity 12 (2007). 